\title{
An Open Question: Is It Rational to Inhibit the mTor-Dependent Pathway as COVID-19 Therapy?
}

\author{
Giuseppe Terrazzano ${ }^{1,2 *}$, Valentina Rubino ${ }^{1}$, Anna Teresa Palatucci ${ }^{2}$, Angela Giovazzino ${ }^{2}$, \\ Flavia Carriero $^{2}$ and Giuseppina Ruggiero ${ }^{2}$
}

${ }^{1}$ Department of Translational Medical Sciences, University of Naples Federico II, Naples, Italy, ${ }^{2}$ Department of Science,

University of Basilicata, Potenza, Italy

Keywords: COVID-19, hyper-activation, immune-regulation, mTOR, Everolimus

\section{OPEN ACCESS}

Edited by:

Paola Patrignani,

University of Studies G. d'Annunzio

Chieti and Pescara, Italy

Reviewed by:

Annalisa Trenti,

University of Padova, Italy

Melania Dovizio,

University of Studies G. d'Annunzio

Chieti and Pescara, Italy

*Correspondence:

Giuseppe Terrazzano

giuseppe.terrazzano@unibas.it

Specialty section:

This article was submitted to Inflammation Pharmacology,

a section of the journal

Frontiers in Pharmacology

Received: 04 May 2020

Accepted: 25 May 2020

Published: 29 May 2020

Citation:

Terrazzano G, Rubino V, Palatucci AT, Giovazzino A, Carriero F and Ruggiero G (2020) An Open Question: Is It Rational to Inhibit the mTorDependent Pathway as COVID-19 Therapy?

Front. Pharmacol. 11:856. doi: 10.3389/fphar.2020.00856

\section{INTRODUCTION}

In December 2019, a novel coronavirus infection appeared in China (Wuhan City and Hubei Province), causing the first cases of abnormal severe pneumonia. Since then, the SARS-Cov2 infection has become pandemic and the correlated coronavirus disease (COVID-19) has been showing a plethora of pathophysiological manifestations that do not exclusively reduce COVID-19 to the occurrence of severe acute respiratory distress (Cevik et al., 2020; Chen et al., 2020; Harapan et al., 2020; Ryu and Chun, 2020; Sen et al., 2020).

Although the immunological responses against SARS-Cov2 remain poorly defined (Chen et al., 2020; Li et al., 2020; Nikolich-Zugich et al., 2020; Qin et al., 2020), it is of note that the critical phase of COVID-19 currently appears, at least in some critical pathophysiological aspects, as a sort of autoimmune disease or as immune response hypersensitivity. Consequently, many authors have proposed various therapeutic approaches based on the modulation/inhibition of abnormal immune response in COVID-19 (Askanase et al., 2020; Chen et al., 2020; Geng et al., 2020; Li et al., 2020; Nikolich-Zugich et al., 2020; Piva et al., 2020; Qin et al., 2020; Radbel et al., 2020; Whyte et al., 2020; Ye et al., 2020; Yuki et al., 2020; Zhao, 2020).

Recently, the effects of Tocilizumab administration has seemed to indicate that inhibition of the Interleukin (IL)-6 receptor (IL-6R) may result in the recovery of critical COVID-19 patients (Aziz et al., 2020; Zhang et al., 2020) in the advanced post-alveolitic phase, when extensive pulmonary fibrosis is accompanied by a diffuse interstitial inflammation apparently sustained by a described exacerbated cytokine storm (Askanase et al., 2020; Chen et al., 2020; Geng et al., 2020; Li et al., 2020; Nikolich-Zugich et al., 2020; Piva et al., 2020; Qin et al., 2020; Radbel et al., 2020; Whyte et al., 2020; Ye et al., 2020; Yuki et al., 2020; Zhao, 2020). Such evidence highlights the critical relevance of controlling the IL-6/IL-6R pro-inflammatory pathway in the pathophysiology of COVID-19 in order to mitigate the adverse immune response that is a determinant of the most serious and undesirable phase of SARS-Cov2 infection (Whyte et al., 2020; Yuki et al., 2020; Zhao, 2020). In particular, hyper-reactivity was described as a major feature of the critical phase of COVID-19, broadly due to the hyperacute inflammatory context that leads to pulmonary interstitial disease and severe acute respiratory distress (Askanase et al., 2020; Chen et al., 2020; Geng et al., 2020; Li et al., 2020; Nikolich-Zugich et al., 2020; Piva et al., 2020; Qin et al., 2020; Radbel et al., 2020; Whyte et al., 2020; Ye et al., 2020; Yuki et al., 2020; Zhao, 2020). 


\section{THE OBSERVED HYPERACTIVATION OF THE IMMUNE RESPONSE IN COVID-19 AND THE HYPOTHESIS OF AN EVEROLIMUS APPROACH TO INHIBIT THE MTOR-DEPENDENT PATHWAY}

Taken as a whole, several datasets produced so far on COVID-19 suggest a central role for immune response dysregulation in the pathophysiological features occurring in the severe forms of this infectious disease (Askanase et al., 2020; Chen et al., 2020; Geng et al., 2020; Li et al., 2020; Nikolich-Zugich et al., 2020; Piva et al., 2020; Qin et al., 2020; Radbel et al., 2020; Whyte et al., 2020; Ye et al., 2020; Yuki et al., 2020; Zhao, 2020). Therefore, it appears legitimate to consider the use of therapies aimed at controlling/ inhibiting the immune response by using drugs acting on immune homeostasis (Askanase et al., 2020; Chen et al., 2020; Geng et al., 2020; Li et al., 2020; Nikolich-Zugich et al., 2020; Piva et al., 2020; Qin et al., 2020; Radbel et al., 2020; Whyte et al., 2020; Ye et al., 2020; Yuki et al., 2020; Zhao, 2020).

In the context of immune response regulation, of great relevance is the role of regulatory $\mathrm{T}$ cells (Tregs), which are a subpopulation of $\mathrm{CD}^{+} \mathrm{T}$ lymphocytes involved in the control of immunological self-tolerance and in the maintenance of immune homeostasis (Sakaguchi, 2005; Strauss et al., 2007; Procaccini et al., 2010). In addition, the intracellular mammalian Target Of Rapamycin (mTOR) molecule plays a key role in immune-regulation and immune-tolerance control pathways (Wullschleger et al., 2006; Dowling et al., 2010; Fasolo and Sessa, 2014; Chen et al., 2019).

mTor is the main intracellular nutrient sensor and, a serinethreonine kinase, its activity acts to regulate the cell cycle and growth by "sensing" the extracellular energy state given by amino acids, glucose, growth factors, and hormones (Dunlop and Tee, 2009; Laplante and Sabatini, 2009; Dowling et al., 2010; Galgani et al, 2010). It favors cellular metabolism and growth when conditions are favorable or catabolic processes when conditions are unfavorable. mTOR is present in two different multi-protein complexes. 1) mTOR Complex 1 (mTORC1) (Dunlop and Tee, 2009; Laplante and Sabatini, 2009; Dowling et al., 2010), formed by the association of mTOR with Raptor, GBL, PRAS40, and Deptor. Briefly, a high level of ATP, growth factors, and hormones activate mTORC1. Conversely, low ATP levels and the absence of growth factors inhibit mTORC1. Activated mTORC1, through target phosphorylation (such as p70 S6 kinase), induces metabolic effects such as mRNA translation, ribosome biogenesis, protein synthesis, mitochondrial metabolism, and adipogenesis. 2) mTOR Complex 2 (mTORC2) (Dunlop and Tee, 2009; Laplante and Sabatini, 2009; Dowling et al., 2010), composed of mTOR, Rictor, G $\beta$ L, Sin1, PRR5/Protor-1, and Deptor. mTORC2 promotes cell survival (via Akt), cytoskeleton (via PKC), ion transport, and cell growth (via SGK1).

Aberrant signaling of mTOR is involved in many pathological states, such as cancer, cardiovascular disease, inflammation, autoimmunity, and metabolic disorders (Dowling et al., 2010; Procaccini et al., 2010; Fasolo and Sessa, 2014; Chen et al., 2019).

Rapamycin is the prototypic mTOR-inhibitor drug (Albert et al., 2010). Several analogs of Rapamycin have been synthetized for effective therapeutic use (Albert et al., 2010). In this context, Everolimus is a promising second-generation rapamycin derivative in terms of adverse effect management (Kaplan et al., 2014). Since it is a specific inhibitor of mTORC1, the drug down-modulates mRNA translation, ribosome biogenesis, protein synthesis, mitochondrial metabolism, and adipogenesis (Dunlop and Tee, 2009; Laplante and Sabatini, 2009; Dowling et al., 2010). In this regard, mTORC1 inhibition by Everolimus is effectively used in several transplantation therapies and in a broad range of disease therapies or anti-tumoral approaches (Hernández et al., 2011; Fasolo and Sessa, 2014). Classic mTOR inhibition by Rapamycin exerts opposite effects on conventional $\mathrm{T}$ lymphocytes and on Tregs, and the differential impact is likely dependent on the intracellular state in the two cellular subpopulations (Strauss et al., 2007; Procaccini et al., 2010). Briefly, cell growth of conventional T lymphocytes is inhibited by Rapamycin, while such a drug expands Tregs proliferation (Strauss et al., 2007; Procaccini et al., 2010). Therefore, mTOR inhibition by drugs - and, specifically, the Everolimus-mediated mTORC1 inhibition - may also assert this differential effect in COVID-19: a reduction in proliferation of conventional $\mathrm{T}$ lymphocytes, which could mitigate the cytokine storm, and preserved Treg growth and activity, which could reduce the hyper-reactivity in the critical phase of the disease (Figure 1).

The inhibition of mTOR may prevent the immune hyperactivation of the signal via the STAT3 pathway that, in turn, has been described to increase the expression of receptors for IL-6 and IL-6 production itself (Wullschleger et al., 2006; Yu et al., 2007; Dowling et al., 2010; Fasolo and Sessa, 2014; Chen et al., 2019). The inhibition of mToR by Everolimus also results in a complex favorable modulation of cancer and of immune response interplay (Hernández et al., 2011; Fasolo and Sessa, 2014; Sabbatini et al., 2015; Johnson et al., 2018).

Our research group observed (Sabbatini et al., 2015) that, in kidney transplant patients undergoing conversion from the calcineurin inhibitor Ciclosporin to Everolimus, the obtained balanced mTOR inhibiting effect was able to guarantee more controlled and specific immunosuppression than calcineurin inhibitors, for example by maintaining high and qualitatively effective levels of Tregs, inhibiting the secretion of proinflammatory IL-17 and IFN- $\gamma$ cytokines, and reducing the hyper-activation of CD8 T cells in kidney post-transplantation. Such aspects could be of some relevance also in avoiding the occurrence of pulmonary fibrosis in COVID-19 (Figure 1), which could be due to the cytokine storm and immune response hyper-activation (Askanase et al., 2020; Chen et al., 2020; Geng et al., 2020; Li et al., 2020; Nikolich-Zugich et al., 2020; Piva et al., 2020; Qin et al., 2020; Radbel et al., 2020; Whyte et al., 2020; Ye et al., 2020; Yuki et al., 2020; Zhao, 2020).

Moreover, Everolimus has surprisingly been associated with the reduction of viral replication of $\mathrm{CMV}, \mathrm{BKV}$, and $\mathrm{HCV}$ post- 


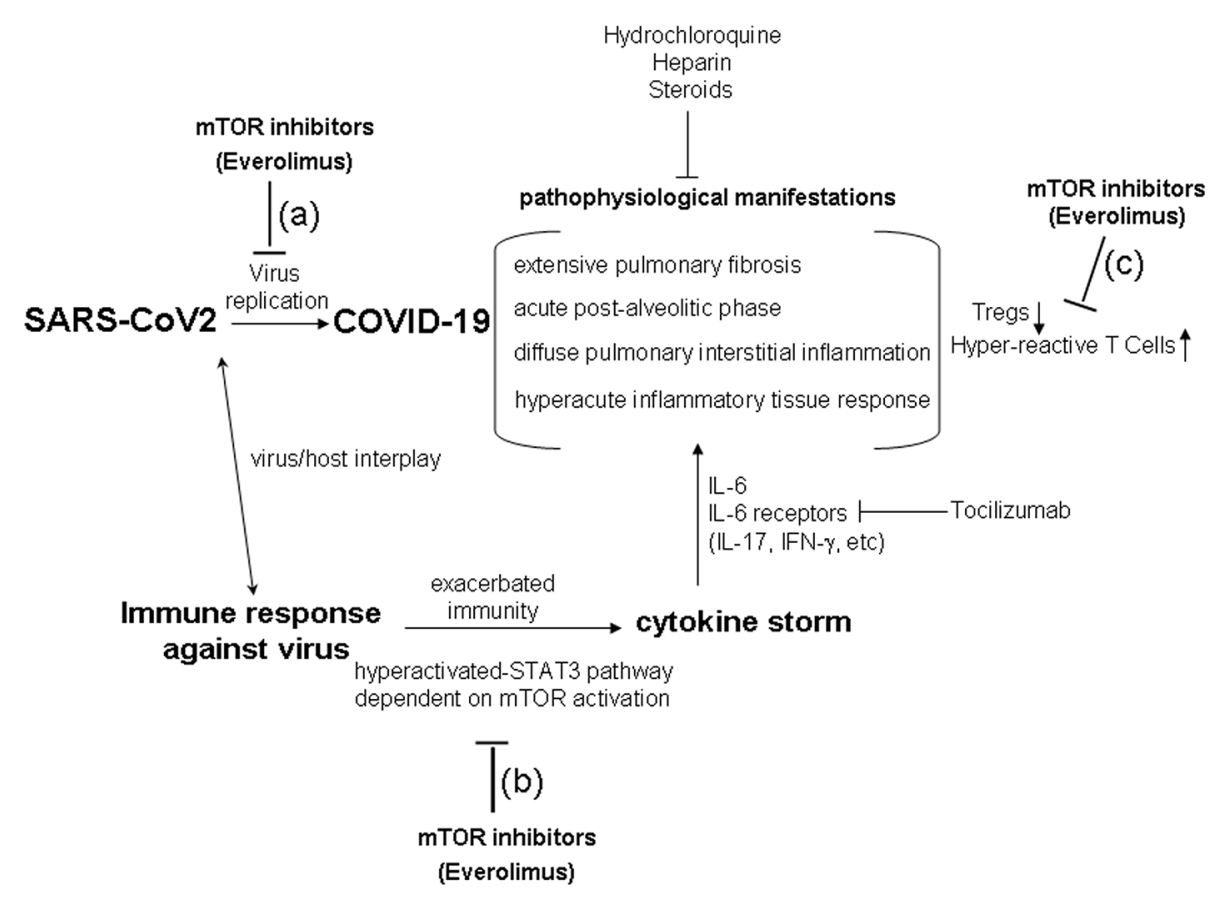

FIGURE 1 | The hypothesis of a therapy using mTOR inhibitors (Everolimus) in COVID-19. The critical phases of COVID-19 show pathophysiological aspects resembling a sort of immune response hypersensitivity, mediated by exacerbated immune mechanism and cytokine storm. mTOR inhibition (i.e., by Everolimus) could act in reducing SARS-CoV2 replication (A), in inhibiting the cytokine storm dependent on the hyper-activated-STAT3 pathway (B), in contrasting Treg downregulation, and in reducing conventional hyper-reactive T cells (C) in COVID-19. Current step-use of Tocilizumab, Hydrochloroquine, Heparin, and Steroids is reported in the figure.

transplantation and in cancer patients (Garofalo et al., 2019; Nanmoku et al., 2019; Tan et al., 2019), although the specific drug mechanism has never been definitively clarified. In this regard, the combination of antiviral drugs like leflunomide and fluoroquinolones/Everolimus should favor BKV viremia clearance (Garofalo et al., 2019), and the conversion from conventional immunosuppressant drugs to Everolimus appears to induce the remission of EBV-related lymphoproliferative disorder in kidney transplantation patients (Nanmoku et al., 2019). Moreover, Everolimus has been described to effectively inhibit in vitro CMV replication in infected cells (Tan et al., 2019).

\section{DISCUSSION}

The question to be answered is whether a therapy that uses Everolimus in COVID-19 could reduce the pathophysiological hyperactivation of the immune response in the lung and other organs described as extensively degenerated by inflammation upon infection with this coronavirus (Chen et al., 2020; Li et al., 2020; Nikolich-Zugich et al., 2020; Piva et al., 2020; Qin et al., 2020; Radbel et al., 2020; Whyte et al., 2020; Ye et al., 2020; Yuki et al., 2020; Zhao, 2020).

It is certainly a gamble to administer a potentially immunosuppressive drug in a viral infection, and therefore
Everolimus should probably be used at doses close to those used in anti-tumor therapy to avoid adverse effects dependent on the immune-depression emerging at higher doses. As referred to in the previous paragraph, Everolimus may inhibit conventional $\mathrm{T}$ lymphocytes and may maintain Treg functions to reduce hyper-reactivity in COVID-19 (Figure 1). However, Everolimus could be administered together with current therapeutic approaches, particularly in the critical phase of SARS-Cov2 infection (Askanase et al., 2020; Chen et al., 2020; Geng et al., 2020; Li et al., 2020; Nikolich-Zugich et al., 2020; Piva et al., 2020; Qin et al., 2020; Radbel et al., 2020; Whyte et al., 2020; Ye et al., 2020; Yuki et al., 2020; Zhao, 2020). Indeed, since hyper-reactivity is one of the determinants of COVID-19 critical phase, Everolimus could be utilized for the same rational use as Tocilizumab, Hydrochloroquine, Heparin, and Steroids in the intensive therapy of COVID-19 (Askanase et al., 2020; Chen et al., 2020; Geng et al., 2020; Li et al., 2020; Nikolich-Zugich et al., 2020; Piva et al., 2020; Qin et al., 2020; Radbel et al., 2020; Whyte et al., 2020; Ye et al., 2020; Yuki et al., 2020; Zhao, 2020). Moreover, the putative anti-replicative effect of Everolimus in controlling viral spread could also be promising in SARS-CoV2 infection (Figure 1) on the basis of its ability to reduce mRNA translation, ribosome biogenesis, protein synthesis, mitochondrial metabolism, and viral replication (Dunlop and Tee, 2009; Laplante and Sabatini, 2009; Dowling et al., 2010; Garofalo et al., 2019; Nanmoku et al., 2019; Tan et al., 2019). 
Honestly, the authors of this short opinion do not have an answer; they aim only to propose to clinicians the hypothesis of modulating the immune response by acting on mTor, as a main immune-regulating key molecule, in the complex disease of SARS-CoV2 infection.

\section{AUTHOR CONTRIBUTIONS}

GR and GT contributed equally, conceptualized the paper, and wrote the manuscript. VR, AP, AG, and FC contributed to the manuscript and read, edited, and approved the submitted version.

\section{REFERENCES}

Albert, S., Serova, M., Dreyer, C., Sablin, M. P., Faivre, S., and Raymond, E. (2010). New inhibitors of the mammalian target of rapamycin signaling pathway for cancer. Expert. Opin. Investig. Drugs 19, 919-930. doi: 10.1517/ 13543784.2010.49912

Askanase, A. D., Khalili, L., and Buyon, J. P. (2020). Thoughts on COVID-19 and autoimmune diseases. Lupus Sci. Med. 3, 7. doi: 10.1136/lupus-2020-000396

Aziz, M., Fatima, R., and Assaly, R. (2020). Elevated Interleukin-6 and Severe COVID-19: A Meta-Analysis. J. Med. Virol. doi: 10.1002/jmv.25948

Cevik, M., Bamford, C., and Ho, A. (2020). COVID-19 pandemic - A focused review for clinicians. Clin. Microbiol. Infect. doi: 10.1016/j.cmi.2020.04.023; pii: S1198-743X(20)30231-7.

Chen, Y., Colello, J., Jarjour, W., and Zheng, S. G. (2019). Cellular Metabolic Regulation in the Differentiation and Function of Regulatory T Cells. Cells. 21, 8. doi: 10.3390/cells8020188

Chen, Y., Liu, Q., and Guo, D. (2020). Emerging coronaviruses: Genome structure, replication, and pathogenesis. J. Med. Virol. 92, 418-423. doi: 10.1002/ jmv. 25681

Dowling, R. J., Topisirovic, I., Fonseca, B. D., and Sonenberg, N. (2010). Dissecting the role of mTOR: lessons from mTOR inhibitors. Biochim. Biophys. Acta 1804, 433-439. doi: 10.1016/j.bbapap.2009.12.001

Dunlop, E. A., and Tee, A. R. (2009). Mammalian target of rapamycin complex 1: signalling inputs, substrates and feedback mechanisms. Cell. Signal. 21, 827835. doi: 10.1016/j.cellsig.2009.01.012

Fasolo, A., and Sessa, C. (2014). Targeting mTOR pathways in human malignancies. Curr. Pharm. Des. 2012 18, 2766-2777. doi: 10.2174/ 138161212800626210

Galgani, M., Procaccini, C., De Rosa, V., Carbone, F., Chieffi, P., La Cava, A., et al. (2010). Leptin modulates the survival of autoreactive CD4+ T cells through the nutrient/energy-sensing mammalian target of rapamycin signaling pathway. $J$. Immunol. 185, 7474-7479. doi: 10.4049/jimmunol.1001674

Garofalo, M., Pisani, F., Lai, Q., Montali, F., Nudo, F., Gaeta, A., et al. (2019). Viremia Negativization After BK Virus Infection in Kidney Transplantation: A National Bicentric Study. Transplant. Proc. 51, 2936-2938. doi: 10.1016/ j.transproceed.2019.04.091

Geng, Y.-J., Wei, Z.-W., Qian, H.-Y., Huang, J., Lodato, R., and Castriotta, R. J. (2020). Pathophysiological Characteristics and Therapeutic Approaches for Pulmonary Injury and Cardiovascular Complications of Coronavirus Disease 2019 Cardiovasc. Pathol. doi: 10.1016/j.carpath.2020.107228. 107228.

Harapan, H., Itoh, N., Yufika, A., Winardi, W., Keam, S., Te, H., et al. (2020). Coronavirus disease 2019 (COVID-19): A literature review. J. Infect. Public Health. doi: 10.1016/j.jiph.2020.03.019. pii: S1876-0341(20)30432-9.

Hernández, D., Martínez, D., Gutiérrez, E., López, V., Gutiérrez, C., García, P., et al. (2011). Clinical evidence on the use of anti-mTOR drugs in renal transplantation. Nefrologia. 31, 27-34. doi: 10.3265/Nefrologia. pre2010.Jul.10512

Johnson, D. E., O’Keefe, R. A., and Grandis, J. R. (2018). Targeting the il-6/jak/ stat3 signalling axis in cancer. Nat. Rev. Clin. Oncol. 15, 234-248. doi: 10.1111/ nyas. 12122

Kaplan, B., Qazi, Y., and Wellen, J. R. (2014). Strategies for the management of adverse events associated with mTOR inhibitors. Transplant. Rev. (Orlando). 28, 126-133. doi: 10.1016/j.trre.2014.03.002

Laplante, M., and Sabatini, D. M. (2009). mTOR signaling at a glance. J. Cell. Sci. 122 (Pt 20), 3589-3594. doi: 10.1242/jcs.051011

Li, G., Fan, Y., Lai, Y., Han, T., Li, Z., Zhou, P., et al. (2020). Coronavirus infections and immune responses. J. Med. Virol. 92, 424-432. doi: 10.1002/jmv.25685

Nanmoku, K., Shinzato, T., Kubo, T., Shimizu, T., and Yagisawa, T. (2019). Remission of Epstein-Barr virus-positive post-transplant lymphoproliferative disorder by conversion to everolimus in a kidney transplant recipient. Transpl. Infect. Dis. 21, e13116. doi: 10.1111/tid.13116

Nikolich-Zugich, J., Knox, K. S., Rios, C. T., Natt, B., Bhattacharya, D., and Fain, M. J. (2020). SARS-CoV-2 and COVID-19 in older adults: what we may expect regarding pathogenesis, immune responses, and outcomes. Geroscience. 42, 505-514. doi: 10.1007/s11357-020-00186-0

Piva, S., Filippini, M., Turla, F., Cattaneo, S., Margola, A., De Fulviis, S., et al. (2020). Clinical presentation and initial management critically ill patients with severe acute respiratory syndrome coronavirus 2 (SARS-CoV-2) infection in Brescia, Italy. J. Crit. Care 58, 29-33. doi: 10.1016/j.jcrc.2020.04.004

Procaccini, C., De Rosa, V., Galgani, M., Abanni, L., Calì, G., Porcellini, A., et al. (2010). An oscillatory switch in mTOR kinase activity sets regulatory T cell responsiveness. Immunity. 33, 929-941. doi: 10.1016/j.immuni.2010.11.024

Qin, C., Zhou, L., Hu, Z., Zhang, S., Yang, S., Tao, Y., et al. (2020). Dysregulation of immune response in patients with COVID-19 in Wuhan, China. Clin. Infect. Dis. doi: 10.1093/cid/ciaa248. pii: ciaa248.

Radbel, J., Narayanan, N., and Bhatt, P. J. (2020). Use of tocilizumab for COVID19 infection-induced cytokine release syndrome: A cautionary case report. Chest. pii: S0012-3692(20)30764-9. doi: 10.1016/j.chest.2020.04.024

Ryu, S., and Chun, B. C. (2020). Korean Society of Epidemiology 2019-nCoV Task Force Team. interim Rev. Epidemiol. Character. 2019 novel Coronavirus Epidemiol. Health 42, e2020006. doi: 10.4178/epih.e2020006

Sabbatini, M., Ruggiero, G., Palatucci, A. T., Rubino, V., Federico, S., Giovazzino, A., et al. (2015). Oscillatory mTOR inhibition and Treg increase in kidney transplantation. Clin. Exp. Immunol. 182, 230-240. doi: 10.1111/cei.12669

Sakaguchi, S. (2005). Naturally arising Foxp3-expressing $\mathrm{CD} 25^{+} \mathrm{CD} 4^{+}$regulatory $\mathrm{T}$ cells in immunological tolerance to self and nonself. Nat. Immunol. 6, 345352. doi: $10.1038 /$ ni1 178

Sen, S., Anand, K. B., Karade, S., and Gupta, R. M. (2020). Coronaviruses: origin and evolution. Med. J. Armed Forces India. 76, 136-141. doi: 10.1016/ j.mjafi.2020.04.008

Strauss, L., Whiteside, T. L., Knights, A., Bergmann, C., Knuth, A., and Zippelius, A. (2007). Selective survival of naturally occurring human CD4+CD25+Foxp3+ regulatory $\mathrm{T}$ cells cultured with rapamycin. J. Immunol. 178, 320-329. doi: 10.4049/jimmunol.178.1.320

Tan, L., Sato, N., Shiraki, A., Yanagita, M., Yoshida, Y., Takemura, Y., et al. (2019). Everolimus delayed and suppressed cytomegalovirus DNA synthesis, spread of the infection, and alleviated cytomegalovirus infection. Antiviral Res. 162, 3038. doi: 10.1016/j.antiviral.2018.12.004

Whyte, C. S., Morrow, G. B., Mitchell, J. L., Chowdary, P., and Mutch, N. J. (2020). Fibrinolytic abnormalities in acute respiratory distress syndrome (ARDS) and versatility of thrombolytic drugs to treat COVID-19. J. Thromb. Haemost. doi: $10.1111 /$ jth.14872

Wullschleger, S., Loewith, R., and Hall, M. N. (2006). TOR signalling in growth and metabolism. Cell. 124, 471-484. doi: 10.1016/j.cell.2006.01.016

Ye, Q., Wang, B., and Mao, J. (2020). The pathogenesis and treatment of the 'Cytokine Storm' in COVID-19. J. Infect. doi: 10.1016/j.jinf.2020.03.037. pii: S0163-4453(20)30165-1. 
Yu, H., Kortylewski, M., and Pardoll, D. (2007). Crosstalk between cancer and immune cells: Role of stat 3 in the tumour microenvironment. Nat. Rev. Immunol. 7, 41-51. doi: 10.1038/nri1995

Yuki, K., Fujiogi, M., and Koutsogiannaki, S. (2020). COVID-19 pathophysiology: A review. Clin. Immunol. Apr20, 108427. doi: 10.1016/j.clim.2020.10842

Zhang, S., Li, L., Shen, A., Chen, Y., and Qi, Z. (2020). Rational Use of Tocilizumab in the Treatment of Novel Coronavirus Pneumonia. Clin. Drug Investig. doi: 10.1007/s40261-020-00917-3

Zhao, M. (2020). Cytokine storm and immunomodulatory therapy in COVID-19: Role of chloroquine and anti-IL-6 monoclonal antibodies. Int. J. Antimicrob. Agents. 16, 105982. doi: 10.1016/j.ijantimicag.2020.105982
Conflict of Interest: The authors declare that the research was conducted in the absence of any commercial or financial relationships that could be construed as a potential conflict of interest.

Copyright $\odot 2020$ Terrazzano, Rubino, Palatucci, Giovazzino, Carriero and Ruggiero. This is an open-access article distributed under the terms of the Creative Commons Attribution License (CC BY). The use, distribution or reproduction in other forums is permitted, provided the original author(s) and the copyright owner(s) are credited and that the original publication in this journal is cited, in accordance with accepted academic practice. No use, distribution or reproduction is permitted which does not comply with these terms. 